\title{
CONTROL DE UN SISTEMA DE VENTILACIÓN MEDIANTE LoopWin APLICANDO DIVERSOS MÉTODOS
}

\author{
F. Javier García Ruiz \\ ITAP, Universidad de Valladolid, 47011, Valladolid, javgar@eii.uva.es \\ Eduardo J. Moya de la Torre, Alfonso Poncela \\ ITAP, Universidad de Valladolid, 47011, Valladolid \{ edumoy, poncela\}@eii.uva.es \\ Alexander Curiel Robles \\ Jordán Jiménez Rodríguez
}

\begin{abstract}
El objetivo de este trabajo es diseñar e implementar un controlador para un sistema de ventilación, el cual debe encontrarse en el interior de un maletín para mostrar su funcionamiento con fines puramente didácticos.

A grandes rasgos, el sistema se compone de una resistencia alojada en el interior de un tubo de aluminio, la cual irradiará calor. En un extremo del tubo se sitúa un sensor de temperatura para conocer el valor de esta. En el extremo opuesto un ventilador. La temperatura deberá mantenerse regulada de la mejor forma posible según el valor de consigna que se haya seleccionado. Para conseguir este fin se usará un controlador comercial que pueda encontrarse habitualmente en la industria y que regulará tanto la potencia de la resistencia, como la velocidad del ventilador.
\end{abstract}

Palabras Clave: Sensor de temperatura, regulador, control PID.

\section{INTRODUCCIÓN}

En el marco de las asignaturas de control que se imparten sobre todos en el Grado de Electrónica Industrial y Automática (GEIA) en la Universidad de Valladolid, se desea realizar un equipo de control que pueda utilizarse como equipo de prácticas, con un manejo simple y fácilmente transportable. El sistema elegido será un sistema de control de temperatura en un túnel con ventilación forzada.

Los pasos a seguir serán:

- Diseño del sistema a realizar, elección de componentes.

- Conexionado de los componentes.

- Diseño del controlador a partir de las funciones de sintonizado que ofrece el controlador de tipo industrial HS-7300 (DESIN Instruments) [2] utilizando el software Loopwin [3].

- El equipo debe ser susceptible de transportarlo de forma sencilla mediante un maletín, por lo que debe poder desplegarse o recogerse según las necesidades.

El presente artículo se organiza de la siguiente manera: Después de una breve introducción en la sección 2 se describe el equipo utilizado tanto en su parte software como hardware con todos sus componentes adicionales, en la sección 3 se muestra el funcionamiento del sistema con las condiciones que se pretende cumplir, en la sección 4 se presenta el diseño del controlador PID a través de diversos métodos y en diversas condiciones. Finalmente, en la sección 5 se ofrece un análisis y comparativa de los distintos resultados obtenidos, en la sección 6 se ofrecen algunas de las principales conclusiones obtenidas para la utilización del equipo de prácticas en el Grado de Electrónica Industrial y Automática (GEIA) en la Universidad de Valladolid, y las referencias utilizadas.

\section{CONSTITUCIÓN DEL EQUIPO}

\subsection{SOFTWARE}

En esta sección se describe el software utilizado y los drivers necesarios.

\subsubsection{LOOPWIN}

En primer lugar, decir que LoopWin es un software del fabricante DESIN Instruments que permite programar, configurar y parametrizar el controlador. El software LoopWin dispone de un editor gráfico que permite realizar tanto On Line como Off Line arquitecturas especiales, presentándolas en su tablero de trabajo.

\subsubsection{Drivers para la comunicación con el controlador HS-7300}

Para la utilización del software hace falta una serie de drivers para la comunicación con el controlador HS7300 utilizado, estos son un conjunto de 
instrucciones que permiten la comunicación entre el controlador HS-7300 y un ordenador a través del convertidor PD10.

\subsection{HARDWARE}

En la Figura 1, se muestra el controlador HS-7300 con el que se ha trabajado.
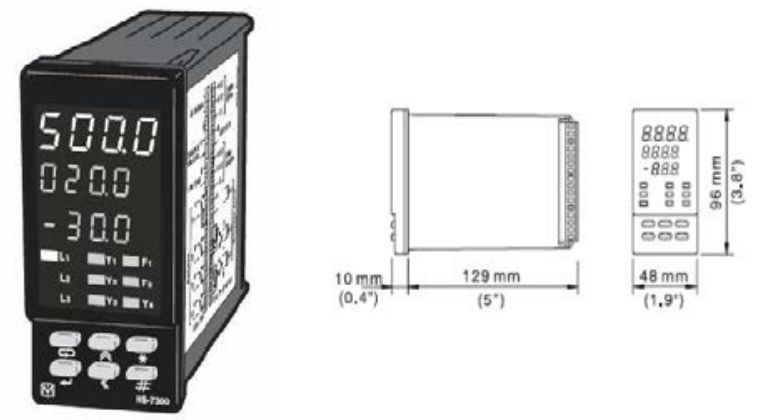

Figura 1: Controlador HS-7300

\subsubsection{Controlador HS-7300}

El controlador HS-7300 pertenece a la serie HS-7000 de equipos de control para panel del fabricante Desin Instruments. Por sus prestaciones universales, exactitud y rapidez de respuesta está encuadrado en la parte alta de gama de los instrumentos de su clase. La serie HS-7000 ha sido desarrollada en tecnología PAC, y dispone de programación abierta con macro bloques de función que, interconectándolos, permiten realizar funciones complejas.

Por ello, es posible realizar infinidad de arquitecturas, estructuras y estrategias de medida y control, prácticamente sin límite.

\subsubsection{Convertidor PD10}

Es un dispositivo que transmite datos desde la interfaz USB a la interfaz industrial RS-485. Por tanto, es utilizado para la transmisión y recepción de datos entre el controlador y el ordenador.

\subsubsection{Servoamplificador}

Se utiliza un servoamplificador como el de la Figura 2.

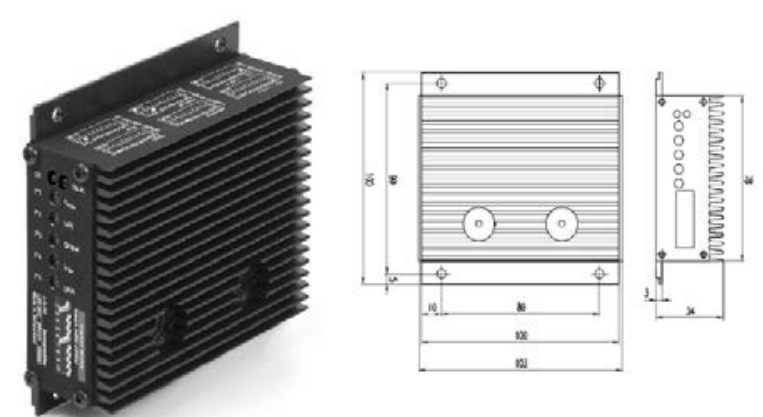

Figura 2: Servoamplificador LSC 30/2
El LSC 30/2 del fabricante Maxon[3] es un servoamplificador lineal de 4 cuadrantes para el control de motores de corriente continua.

Ofrece varios modos de funcionamiento dependiendo de la acción que se requiera. En el sistema que se trata sirve para regular el voltaje que le llega al motor del ventilador en función de una señal de control generada por el controlador.

\subsubsection{Otros componentes}

A continuación, pasamos a describir otra serie de componentes utilizados.

\section{- Sensor de temperatura}

El sensor de temperatura elegido es un termopar tipo $\mathrm{K}$ debido a su buena respuesta y facilidad de uso con el controlador.

\section{- Ventilador}

Se utiliza un ventilador axial del fabricante Sunon de 12 VDC y $5 \mathrm{~W}$ para enfriar el sistema y conseguir la temperatura deseada en el sensor de temperatura.

El ventilador no puede estar nunca en reposo, pues se necesita hacer circular una corriente de aire por el interior del tubo para que el calor circule y no tienda a subir, lo que provocaría el excesivo calentamiento del tubo de aluminio en el que se encuentra la resistencia.

\section{- Resistencia}

Para la generación de calor se utiliza una resistencia de calentamiento de $230 \mathrm{~V}$ y $350 \mathrm{~W}$. Esta resistencia está instalada en el interior de un tubo de aluminio.

\section{- Relé}

Se utiliza un mini-relé industrial insertado en un zócalo para cortar el suministro de corriente a la resistencia de calentamiento cuando sea necesario. De esta forma se consigue bajar a temperaturas similares a la temperatura ambiente y enfriar más rápidamente cuando sea necesario.

La necesidad de utilizar un relé en este sistema surgió de la imposibilidad por parte del ventilador de conseguir alcanzar la temperatura de consigna cuando esta es próxima a la temperatura ambiente. Esto se debe al continuo funcionamiento de la resistencia, el cual aumenta la temperatura de forma gradual. Cuando se alcanzan valores de temperatura altos, enfriar el sistema en un tiempo razonable requiere una potencia extra del ventilador. Esto es solucionado con un relé. El relé utilizado es un minirelé industrial del fabricante Finder modelo 46.52 que posee dos contactos de $8 \mathrm{~A}$ y $250 \mathrm{VAC}$, y una 
bobina cuya máxima tensión de excitación son 12VDC. Este minirelé se inserta en un zócalo o interfaz modular del mismo fabricante modelo 4C.02. Este zócalo es el que permite utilizar las conexiones de entrada a la bobina, y las de salida de normalmente abierto (NO) y normalmente cerrado (NC) para conseguir el efecto deseado.

\section{- Tubo de aluminio}

Un tubo de aluminio de aproximadamente $10 \mathrm{~cm}$ de diámetro y $40 \mathrm{~cm}$ de largo para introducir en su interior la resistencia de calentamiento y permitir la canalización de la corriente de aire hacia el sensor de temperatura.

\section{- Circuito conversor}

En el sistema se requiere únicamente una conversión de una señal de corriente (salida de control del controlador) a una señal de tensión (entrada de control del servoamplificador).

\section{- Maletín}

Maletín de aluminio de dimensiones $482 \times 340 \times 205 \mathrm{~mm}$. En su interior se encuentra un mecanismo que permite mostrar el funcionamiento del sistema.

El conjunto final con todos los componentes puede verse en la Fig. 3.

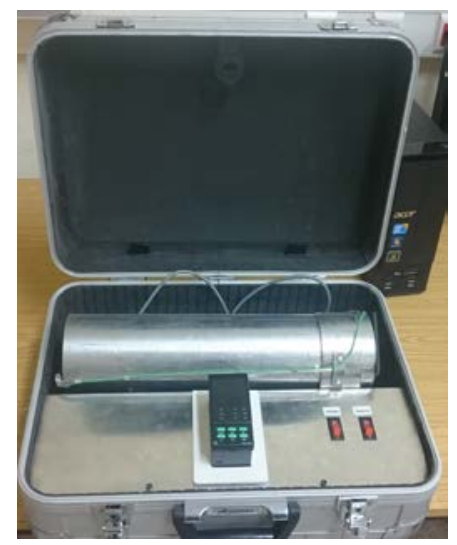

Figura 3: Aspecto final del equipo creado.

\section{FUNCIONAMIENTO}

La forma en la que están relacionados los diferentes elementos de este sistema puede apreciarse de forma genérica en la Fig. 4.

Inicialmente, se introduce una temperatura de consigna (SP). Esta consigna se compara en el controlador con la temperatura detectada por el sensor de temperatura (termopar).

El controlador está configurado en modo de controlador continuo por modulación de corriente. Esto es una forma de control en el que el instrumento produce una acción correctora modulando la corriente 0-20 mA de una salida analógica, que es enviada al servoamplificador, que convierte esa señal en un régimen de giro concreto del ventilador.

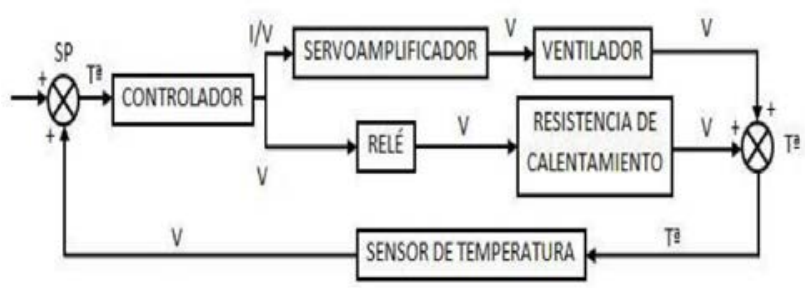

Figura 4: Diagrama de bloques del sistema

En el modo inverso, que es el utilizado, si la variable controlada se desvía del setpoint SP por abajo, el control proporcional corrige disminuyendo la salida de corriente hacia $0 \mathrm{~mA}$ (menor régimen de giro del ventilador implica mayor calentamiento del sistema). Nunca pueden alcanzarse $0 \mathrm{~mA}$ porque implicaría que el ventilador estuviese parado y no circularía el calor. La salida mínima está condicionada por el par motor del ventilador.

Por el contrario, si la variable se desvía por arriba del SP, aumenta la corriente hacia $20 \mathrm{~mA}$ (mayor régimen de giro del ventilador implica menor calentamiento del sistema). En consecuencia, a esto, la variable tiende a centrarse en el setpoint.

La salida de corriente obtenida se convierte en tensión y llega a la entrada de control del servoamplificador. El servoamplificador, configurado adecuadamente como regulador de voltaje, realiza una operación de ganancia y control sobre esta señal recibida, y genera una salida de tensión para el motor del ventilador.

A su vez, la misma señal generada por el controlador se utiliza junto a la salida del servoamplificador para controlar el relé. Según el valor en tanto por ciento de la salida del controlador se permitirá la conexión y desconexión del relé. De esta forma, si detectamos que esta señal es elevada entonces el ventilador estará trabajando a un régimen elevado y será conveniente cortar el flujo de corriente por la resistencia para obtener una respuesta más rápida $\mathrm{o}$ facilitar el enfriamiento para alcanzar temperaturas que de otra forma no serían posibles.

Cuando se necesita cortar el flujo de corriente por la resistencia, la entrada de tensión para cambiar el estado del relé procede de la salida motor que genera el servoamplificador. Es decir, la misma salida que alimenta el motor del ventilador. Esto es así debido a que ambos dispositivos coinciden en su tensión máxima de funcionamiento, lo cual, con unos pocos ajustes en el controlador, nos permite ahorrar una fuente que genere esa tensión.

Tanto la acción llevada a cabo sobre el ventilador 
como sobre la resistencia tendrán un efecto sobre la temperatura detectada ahora por el termopar. Esta temperatura será procesada de nuevo por el controlador como se ha explicado anteriormente.

\section{DISEÑO DEL CONTROLADOR PID}

El controlador HS-7300 proporciona varias herramientas para el cálculo aproximado de los parámetros del PID [1] y su posterior corrección de forma automática. La primera de ellas es el Autosintonizado, el cual tras realizar varias pruebas sobre el sistema ofrece unos valores concretos de los parámetros del PID. La otra herramienta utiliza Lógica Borrosa[6, 8]. Esta permite la corrección del proceso una vez que se observa su comportamiento, pudiendo hacerlo más rápido, más lento o con menor sobrepaso según sea necesario en cada caso.

\subsection{AUTOSINTONIZADO}

Permite calcular de forma automática los parámetros de las acciones PID Inversa o Directa (calor o frío) y Bimodal. Se basa en un algoritmo que aplica a la Salida de Regulación un escalón de amplitud configurable (Pulso Autosintonizado) para que el proceso produzca una respuesta. Con los datos de esa respuesta, el instrumento calculará los valores PID para ese proceso, sustituyendo a los anteriores valores a la finalización del cálculo.

Para iniciar el procedimiento es aconsejable que se cumplan algunas condiciones.

En primer lugar es deseable adecuar el rango (Span) de regulación del instrumento al rango de trabajo del proceso. Esto es recomendable para que una vez realizado el cálculo de la Banda Proporcional, el \% resultante tenga la suficiente resolución para ser aplicado óptimamente.

Por ejemplo, en un instrumento con un Span de regulación de $1200^{\circ} \mathrm{C}$ y un SP de $400^{\circ} \mathrm{C}$. Si el Autosintonizado calcula una banda proporcional de $25^{\mathrm{a}}$, supone aproximadamente una acción proporcional del $2 \%$. Si el rango de trabajo del proceso es hasta $450^{\circ} \mathrm{C}$, adaptar el rango de regulación del Lazo determinado a un valor cercano algo superior, como 0 a $500^{\circ} \mathrm{C}$.

Con este nuevo Span de regulación el mismo Autosintonizado determinará una acción proporcional del $5 \%$ mucho más resolutiva para que los cálculos de PID sean más precisos.

La serie HS-7000 dispone de dos métodos de Autosintonizado:

\subsubsection{Método de Ziegler Nichols}

El más corriente es el del método de Ziegler-Nichols [5] en el que se determina los parámetros PID mediante un cálculo de la frecuencia natural del proceso. Este sistema es apropiado para toda clase de procesos de variación lenta como pueden ser los procesos térmicos, de control de $\mathrm{HR} \%$, etc.

En este método el valor del pulso de Autosintonizado está ajustado de origen al 100\%. Lo que significa que el impulso en escalón dado al proceso es del 100\% de la salida de control. El valor del impulso en escalón no debe ser modificado a no ser que se realice el Autosintonizado por el método 'Relé'.

\subsubsection{Método Relé}

El método del Relé[4, 8] es usado en procesos rápidos, fuertemente alinéales o desconocidos. Se basa en producir un escalón una vez el proceso está regulando cerca de su punto de consigna SP.

El valor del pulso de Autosintonizado ha de ser preseleccionado entre $-20 \%$ y $-50 \%$ si se desea aplicar un escalón negativo, o de $+20 \%$ a $+50 \%$ si se desea un escalón positivo. Eso significa que el impulso aplicado al proceso será en la dirección y \% especificado en Pulse.

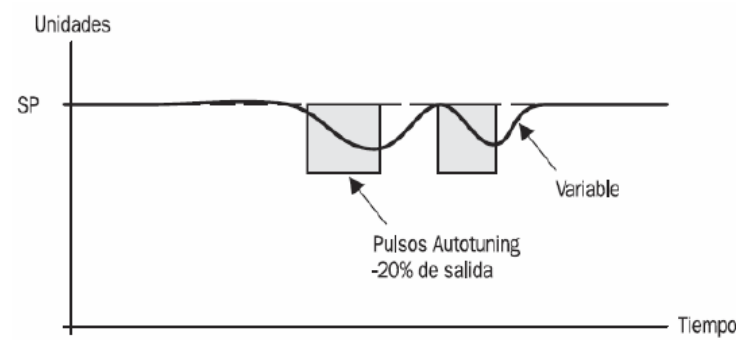

Figura 5: Método relé.

\subsection{FUZZY-LOGIC. OPTIMIZACIÓN DE LA RESPUESTA DE CONTROL}

Permite, siguiendo la lógica humana, ajustar la rapidez, lentitud o sobrepaso (overshoot) de la regulación, optimizando la acción PID para mejorar la respuesta del proceso controlado.

El usuario, evalúa visualmente las necesidades del proceso y corrige la acción de control por medio del Fuzzy-Logic a fin de conseguir más rapidez o lentitud de respuesta, y/o amortiguar el sobrepaso de la variable regulada del proceso.

\subsection{CALCULO FINAL DEL PID}

Para hallar los diferentes valores del PID se procedió de la siguiente forma. Se dividió el rango de temperaturas con el que se trabaja $\left(30^{\circ} \mathrm{C}\right.$ a $\left.70^{\circ} \mathrm{C}\right)$ en porciones de diez grados centígrados. Para cada una de estas porciones se fijó una temperatura intermedia como consigna y se procedió a aplicar la función Autosintonizado para cada una de ellas repetidas veces. De esta forma se obtuvieron unos parámetros del PID promedio para cada rango lo cual fue útil para ver como variaban las necesidades del sistema 
según la temperatura de consigna con la que se trabajaba.

A continuación, se exponen los valores obtenidos para cada porción del rango así como observaciones del comportamiento del sistema.

Estos valores son:

- KPD: Banda proporcional directa

- KID: Tiempo integral directa

- KDD: Tiempo derivada directa

\subsubsection{Rango de $30^{\circ} \mathrm{C}$ a $40^{\circ} \mathrm{C}\left(\right.$ Consigna $\mathrm{SP}: 35^{\circ} \mathrm{C}$ )}

Los valores utilizados son:

- KPD: 6

- KID: 0.3

- KDD: 3

Con la resistencia generando calor durante al menos 2 minutos antes de asignar la consigna, el sistema alcanza $\operatorname{los} 36.5^{\circ} \mathrm{C}$ y tarda 1 minuto y 40 segundos en llegar a $35^{\circ} \mathrm{C}$.

Posteriormente la respuesta es aceptable ya que se mantiene entre $35.1^{\circ} \mathrm{C}$ y $35.4^{\circ} \mathrm{C}$, aunque ocasionalmente bajó hasta los $34.4^{\circ} \mathrm{C}$ y otras veces tomó valores de $1^{\circ} \mathrm{C}$ o $2^{\circ} \mathrm{C}$ por encima de la consigna. Se pudo comprobar que esto se debe principalmente a dos causas. La primera es a la proximidad con la temperatura ambiente, la cual influye notablemente en la temperatura mínima que el sistema es capaz de alcanzar. La segunda es el tiempo de funcionamiento que lleve la resistencia.

Cuanto más tiempo lleve conduciendo esta, más difícil resultará alcanzar temperaturas estables en este rango.

\subsubsection{Rango de $40^{\circ} \mathrm{C} 50^{\circ} \mathrm{C}\left(\right.$ Consigna $\mathrm{SP}: 45^{\circ} \mathrm{C}$ )}

Los valores utilizados son:

- KPD: 8

- KID: 0.2

- KDD: 3

Con la nueva consigna la temperatura asciende hasta $46.9^{\circ} \mathrm{C}$ y tarda 35 segundos en obtener $\operatorname{los} 45^{\circ} \mathrm{C}$. Luego se mantiene entre $44.8^{\circ} \mathrm{C}$ y $45.3^{\circ} \mathrm{C}$ lo cual es aceptable, pero se necesitaría más precisión.

\subsubsection{Rango de $50^{\circ} \mathrm{C} 60^{\circ} \mathrm{C}\left(\right.$ Consigna $\mathrm{SP}: 55^{\circ} \mathrm{C}$ )}

Los valores utilizados son:

- KPD: 12

- KID: 0.2

- KDD: 3

En 31 segundos la temperatura aumenta hasta $57.7^{\circ} \mathrm{C}$ y tarda unos 30 segundos más en llegar a $55^{\circ} \mathrm{C}$.
Finalmente el sistema se mantiene entre $54.7^{\circ} \mathrm{C}$ y $55.3^{\circ} \mathrm{C}$. Es similar al caso anterior.

\subsubsection{Rango de $60^{\circ} \mathrm{C} 70^{\circ} \mathrm{C}\left(\right.$ Consigna $\mathrm{SP}: 65^{\circ} \mathrm{C}$ )}

Los valores utilizados son:

- KPD: 17

- KID: 0.2

- KDD: 3

Se alcanzan $68.1^{\circ} \mathrm{C}$ y el sistema tarda 30 segundos en obtener $65^{\circ} \mathrm{C}$. Al principio el sistema no parece muy estable, pues con las oscilaciones que presenta le cuesta mantenerse fijo en el valor de consigna. Tras 3 minutos y 15 segundos aproximadamente se puede considerar que el sistema se estabiliza y da una respuesta bastante buena de valores entre $65^{\circ} \mathrm{C}$ y $65.2^{\circ} \mathrm{C}$.

Es con diferencia el caso en el que se presenta una mayor estabilidad, pero a cambio de un mayor tiempo con oscilaciones hasta alcanzarla.

\subsubsection{Conclusiones sobre el diseño}

Teniendo en cuenta estos datos se puede ver que:

- el sistema necesita aumentar la banda proporcional directa según aumenta la consigna de temperatura.

- las acciones proporcional y derivativa se mantienen más o menos constantes para diferentes temperaturas.

- el rango de $30^{\circ} \mathrm{C}$ a $40^{\circ} \mathrm{C}$ es el que presenta una mayor inestabilidad.

- el rango de $60^{\circ} \mathrm{C}$ a $70^{\circ} \mathrm{C}$ es el que presenta una mayor estabilidad, pero con una respuesta lenta.

Es por todo esto que se necesita recalcular estos parámetros y encontrar un equilibrio para todo el rango de temperaturas.

La función Fuzzy-Logic ayudará a aumentar la velocidad del sistema, a reducirla y a disminuir los sobrepasos.

Los métodos clásicos de sintonizado de PIDs de Ziegler-Nichols no proporcionaron unas respuestas satisfactorias, siendo estas peores que las generadas con el cálculo automático del PID.

Por tanto, se optó por afinar los parámetros del PID obtenidos de forma automática mediante el método de ensayo y error teniendo como referencia los valores que calcula el PID en las diferentes situaciones del sistema.

Con estos ensayos se pudo observar entre otras cosas que variar la posición del termopar no produce una inestabilidad notoria, pues el sistema vuelve a alcanzar los valores deseados en unos segundos.

Asimismo, en algunos casos (por lo general para temperaturas altas) prescindir de la acción derivativa 
mejoraba la estabilidad, pero no el tiempo de estabilización.

Conseguir que la respuesta del sistema sea igual de buena en todo el rango de temperaturas es bastante complicado, por lo que se intentó encontrar un equilibrio en todas ellas disminuyendo las oscilaciones en la medida de lo posible.

Finalmente se optó por los siguientes valores finales para todo el rango de funcionamiento:

- KPD: 14

- KID: 1

- KDD: 0.1

\section{RESULTADOS}

Cuanta más alta es la temperatura mejor es la respuesta del sistema, pues en el momento en que se puede considerar al sistema estabilizado la temperatura se mantiene muy próxima a la consigna, con variaciones máximas de unos $0.2^{\circ} \mathrm{C}$. Esto puede comprobarse para temperaturas a partir de $\operatorname{los} 50^{\circ} \mathrm{C}$.

Por el contrario, para temperaturas más bajas el sistema es más inestable, pues su respuesta oscila más notoriamente $\mathrm{y}$ se retarda su tiempo de establecimiento, y esto se agrava cuanto mayor sea el tiempo de funcionamiento. Es decir, el calentamiento de la resistencia a lo largo del tiempo afecta más a las temperaturas bajas (considerándose estas entre $30^{\circ} \mathrm{C}$ y $45^{\circ} \mathrm{C}$ ), provocando que el ventilador trabaje a un régimen de giro alto.

Aunque se corta el flujo de corriente por la resistencia cuando el ventilador está trabajando a un $70 \%$ o más, el efecto que esto produce tarda en manifestarse hasta unos pocos minutos si se han alcanzado temperaturas muy elevadas en el sistema. El rango más inestable es entre $40^{\circ} \mathrm{C}$ y $45^{\circ} \mathrm{C}$, con unas variaciones máximas de hasta $0.6^{\circ} \mathrm{C}$ cuando se mantiene lo más estable posible.

Se ha podido observar la fuerte influencia de la temperatura ambiente tanto a la hora de la regulación de los parámetros del PID como en el propio funcionamiento. Esta temperatura ambiente puede visualizarse en el display del controlador, y deberá tenerse en cuenta si se cambian las condiciones de trabajo pues es probable que se necesite un reajuste de los parámetros del sistema.

En casos extremos en los que la temperatura ambiente es elevada, la mínima temperatura en la que puede estabilizarse el sistema se verá afectada aumentando hasta en más de $3^{\circ} \mathrm{C}$.

Generalmente el tiempo de estabilización se considera que es bueno, no demorándose demasiado a no ser que la diferencia de temperaturas sea muy amplia o el sistema se encuentre en una situación límite, como, por ejemplo, introducir una consigna de temperatura muy próxima a la temperatura ambiente, siendo posible incluso no llegarse a alcanzar. El desequilibrio que produce el movimiento del termopar se ve por lo general rápidamente estabilizado.

Para la estabilización de cualquier temperatura existe un sobrepaso, de mayor o menor grado, dependiendo del rango de temperaturas con el que se trabaja y las condiciones en las que se encuentre el sistema.

Para alcanzar temperaturas de consigna más elevadas que a la que se encuentre actualmente el sistema, se producirá un sobrepaso inicial que se verá corregido con el tiempo mediante oscilaciones alrededor de la consigna hasta alcanzar la estabilidad.

De forma similar ocurre para alcanzar temperaturas de consigna por debajo de la que se encuentre el sistema. Existirá igualmente un sobrepaso, haciendo descender la temperatura por debajo de la consigna inicialmente para alcanzar al final un valor estable.

Estos sobre impulsos fueron tratados con la función Fuzzy-Logic que proporciona el controlador.

Se prefirió una respuesta más amortiguada que una respuesta más rápida dentro de una relación de equilibrio entre ambas para no hacer el sistema demasiado lento, pues lo que interesa es alcanzar la temperatura de la forma más precisa posible.

Para mostrar el funcionamiento del sistema control se realizó la siguiente experiencia.

Con una temperatura ambiente de $30^{\circ} \mathrm{C}$ se procedió a realizar un ciclo de temperaturas de consigna ascendentes seguido de otro ciclo de temperaturas de consigna descendentes. De esta forma, al ser un funcionamiento continuo podrán apreciarse los valores que se obtendrían en una situación real.

Se comienza dejando calentar la resistencia unos 30 segundos con el ventilador al mínimo régimen de giro y se alcanzan $\operatorname{los} 34^{\circ} \mathrm{C}$. A continuación, se introduce una temperatura de consigna de $35^{\circ} \mathrm{C}$. El comportamiento del sistema en este caso puede observarse en la Fig. 6.

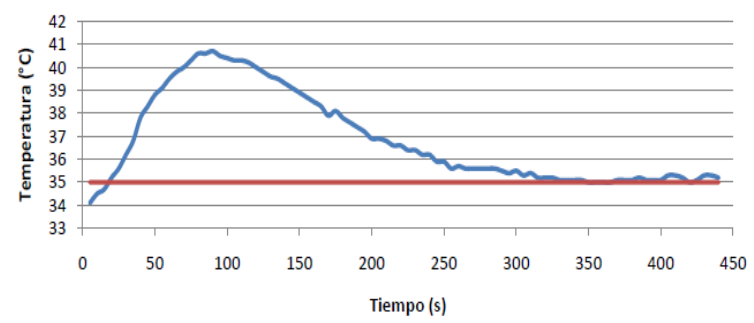

Figura 6: Respuesta para consigna de $35^{\circ}$ (ciclo ascendente)

La respuesta del sistema presenta un sobrepaso con el que se llegan a alcanzar $40.7^{\circ} \mathrm{C}$ a los 90 segundos, el cual es demasiado grande.

Tomando como referencia el valor inicial $\left(34^{\circ} \mathrm{C}\right)$ se puede decir que el tiempo de crecimiento son

18 segundos y su tiempo de establecimiento unos 320 segundos. Como la respuesta presenta una componente oscilatoria se tratará de un sistema subamortiguado de segundo orden. 
Entonces, bajo estas condiciones, la respuesta es lenta, pero se alcanza una buena estabilidad con variaciones de $0.3^{\circ} \mathrm{C}$ como máximo.

Ahora se fija la temperatura de consigna en $45^{\circ} \mathrm{C}$ cuando el termopar está detectando $37.1^{\circ} \mathrm{C}$. Como se ha descrito en un principio, esta temperatura pertenece al rango más inestable del funcionamiento del sistema, por lo que cabe esperar una respuesta oscilatoria con poca estabilidad. El resultado se representa en la Fig. 7.

En este caso se obtiene un sobrepaso que implica alcanzar $\operatorname{los} 47^{\circ} \mathrm{C}$ a los 145 segundos, con una diferencia de $2{ }^{\circ} \mathrm{C}$ respecto de la consigna, por lo que es mucho menor que el sobrepaso del caso inicial.

Tomando como referencia el valor inicial $\left(37.1^{\circ} \mathrm{C}\right)$ se obtiene un tiempo de crecimiento cercano a los 100 segundos. Sin embargo, el tiempo de establecimiento es muy elevado, pues como se ve la repuesta sigue oscilando, aunque cada vez se amortigua más. Esto se debe a que nos encontramos ante un sistema subamortiguado con un coeficiente de amortiguamiento bastante menor que el del caso anterior. Es decir, ahora el coeficiente de amortiguamiento estará más próximo a cero.

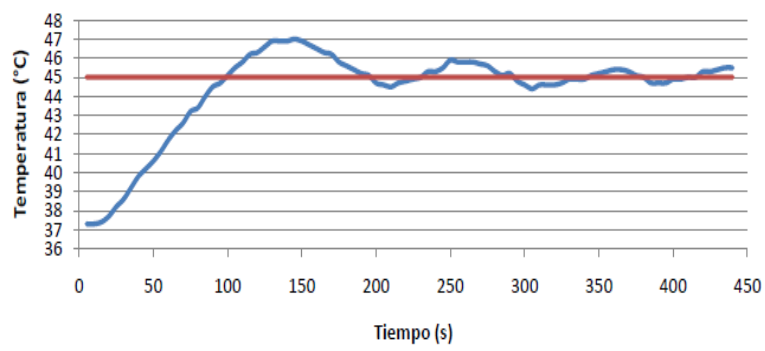

Figura 7: Respuesta para consigna de $45^{\circ}$ (ciclo ascendente)

Por lo tanto en esta situación la respuesta del sistema será oscilante, amortiguándose en el tiempo y con un tiempo de establecimiento que puede superar los 500 segundos.

Modificando el valor de la temperatura de consigna a $55^{\circ} \mathrm{C}$ cuando el sistema se encuentra a $45.5^{\circ} \mathrm{C}$ se obtiene la respuesta representada en la Fig. 8.

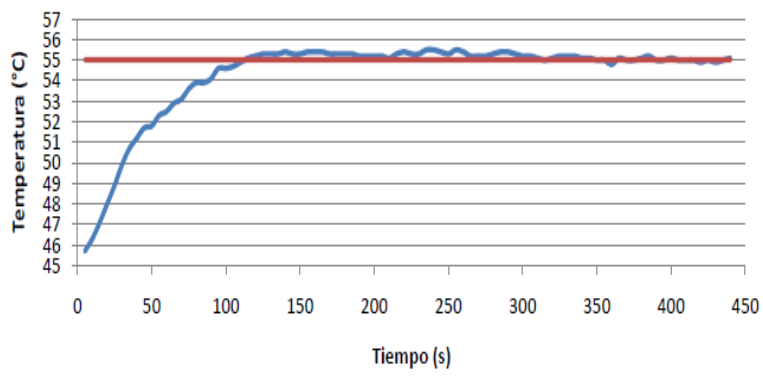

Figura 8: Respuesta para consigna de $55^{\circ}$ (ciclo ascendente)

Contrariamente a los casos anteriores, la respuesta no presenta un sobrepaso y apenas oscila una vez se alcanza la consigna, asimilándose a las respuestas de sistemas de primer orden.

Una vez más tomando como referencia el valor inicial de esta medida $\left(45.5^{\circ} \mathrm{C}\right)$ la respuesta presenta un tiempo de crecimiento cercano de 110 segundos (10 segundos más que en caso anterior). El tiempo de establecimiento podría fijarse en torno a los 200 segundos, pues las variaciones son muy suaves obteniéndose valores aceptables. Siendo más estrictos el tiempo de establecimiento podría fijarse próximo a los 270 segundos.

El sistema podría considerarse por tanto sobreamortiguado o críticamente amortiguado, lo que implica un coeficiente de amortiguamiento muy próximo a la unidad. Es hasta ahora la mejor respuesta tanto en rapidez como en estabilidad, con variaciones mínimas de $0.2^{\circ} \mathrm{C}$. Esto mismo ocurre a temperaturas superiores a $60^{\circ}$.

Comenzando ahora con el ciclo descendente de temperaturas, se asigna de nuevo el valor de $55^{\circ} \mathrm{C}$ a la consigna. Cabe esperar un sobreimpulso negativo como se comentó al inicio del apartado. La respuesta que genera el sistema puede apreciarse en la Fig. 9.

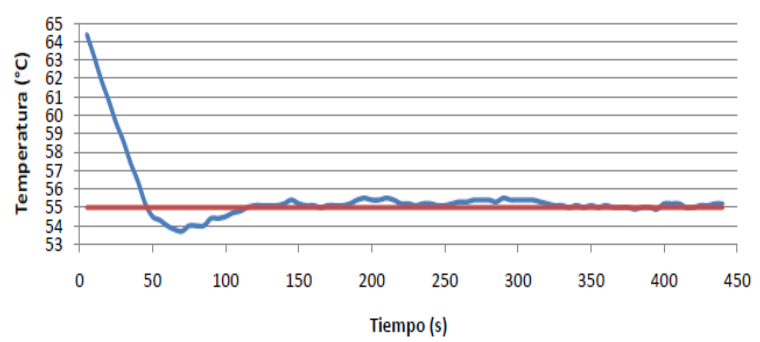

Figura 9: Respuesta para consigna de $55^{\circ}$ (ciclo descendente)

La respuesta presenta un sobrepaso negativo por el que se caracteriza el ciclo descendente. Se llegan a alcanzar los $53.7^{\circ} \mathrm{C}$ lo cual no representa un sobrepaso grande, pues tan solo se desvía $1.4^{\circ} \mathrm{C}$ de la consigna.

Asignando como origen la temperatura anterior del sistema $\left(65^{\circ} \mathrm{C}\right)$ se obtiene un tiempo de crecimiento de 47 segundos y un tiempo de establecimiento de 125 segundos aproximadamente, algo más rápido que con la misma consigna en ciclo ascendente.

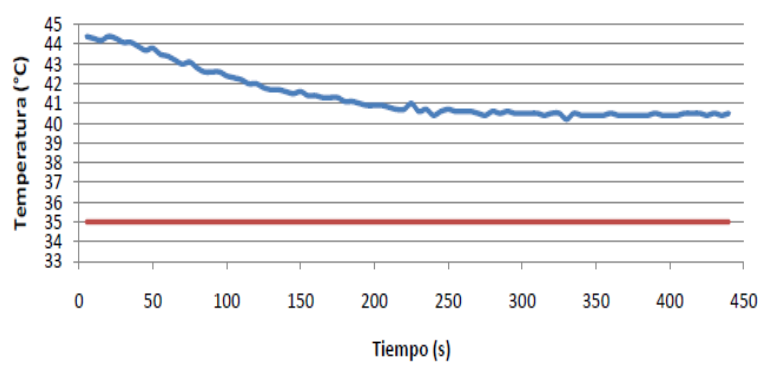

Figura 10: Respuesta para consigna de $35^{\circ}$ (ciclo descendente) 
En vista de estos datos el sistema será subamortiguado, con un coeficiente próximo a uno, lo que reduce el sobrepaso y las oscilaciones a lo largo del tiempo, las cuales son mínimas en este caso. Esto mismo ocurre a temperatura de $45^{\circ}$ donde se obtiene una respuesta oscilatoria.

Para finalizar, se realiza el ensayo con la última consigna que queda por comprobar. Al ajustar este valor a $35^{\circ} \mathrm{C}$ se obtienen los valores de la Fig. 10.

Para interpretar la siguiente respuesta hay que tener en cuenta lo siguiente. En estos momentos el sistema lleva aproximadamente una hora de funcionamiento y la temperatura ambiente ha aumentado de $30^{\circ} \mathrm{C}$ a casi $35^{\circ} \mathrm{C}$. Debido a esto y a que la resistencia tiene una temperatura bastante mayor que al principio, disipar el calor es mucho más costoso. Este es por tanto el problema de establecer valores de consigna próximos a la temperatura ambiente cuando el sistema está en funcionamiento durante largos periodos de tiempo. A simple vista se ve que la respuesta se mantiene más o menos constante a unos $5^{\circ} \mathrm{C}$ de la temperatura de consigna. Tras unos 20 minutos el sistema volvió a sus condiciones de reposo.

\section{APLICACIÓN A LA ENSEÑANZA DE LA REGULACIÓN AUTOMÁTICA}

El equipo está pensado para utilizarlo con los alumnos de la asignatura de Fundamentos de Automática de $2^{\circ}$ curso todos los grados de ingeniería que se imparten en la Escuela de Ingenierías Industriales de la Universidad de Valladolid. Esta asignatura es el primer contacto de los alumnos con la Regulación Automática y al final del curso tienen un capítulo específicamente dedicado al control PID.

Dentro del curso de Fundamentos de Automática los alumnos deben realizar un total de 7 prácticas y realizar un informe de 6 de ellas (la primera es para que se familiaricen con MATLAB). Este equipo está pensado para que sea una de esas 7 prácticas. El trabajo consistiría en hacer una identificación del sistema mediante una serie de entradas escalón y luego diseñar y sintonizar un PID utilizando el controlador incluido en la planta. Previamente en clase de prácticas se les explica el funcionamiento del equipo y se les da una guía con ejemplos de cómo realizar el trabajo.

Los trabajos realizados con las prácticas cuentan un $40 \%$ sobre la nota final, el $60 \%$ restante corresponde al examen final.

\section{CONCLUSIONES}

Se ha construido un sistema de control versátil y adecuado para la docencia de la asignatura de regulación automática en la Escuela de Ingenieros
Industriales de la Universidad de Valladolid. Tiene la ventaja de que es fácilmente transportable y que permite que el alumno entre en contacto con un sistema real, que además utiliza un controlador similar a los que se puede encontrar en su vida profesional.

Se ha demostrado su operabilidad, su fácil transportabilidad y su buen comportamiento. No obstante, existen algunos aspectos del sistema que serían mejorables. La resistencia de calentamiento funciona únicamente en modo todo o nada. Se piensa en un futuro regular la corriente de la resistencia para que varíe el calor que aporta.

\section{Referencias}

[1] K. J. Astrom, Control PID avanzado, España: PRENTICE-HALL, 2009.

[2] D. Instruments. "HS 7300 Controlador Multivariable con cálculo y funciones avanzadas por bloques de función y RS485 MODBUS," 20/05/2016, 2016; http://www.desin.com/pdf/HT-HS7300-es0206-H122-41-2.pdf. (último acceso 13-06-16)

[3] D. Instruments. "Software de programación Loopwin," http://www.desin.com/pdf/DT-LPWes-020718.pdf. (último acceso 13-06-16)

[4] Maxtor. motor. "LSC 30/2 Servo Control 4-QDC Instrucciones de Funcionamiento," 25/05/2016;

http://www.maxonmotor.com/medias/sys_maste r/8803610132510/250521-LSC-30-2-

Instrucciones-de-Funcionamiento-Es.pdf. (último acceso 13-06-16)

[5] R. Kofahl, and R. Isermann, "A Simple Method for Automatic Tuning of PID-Controllers based on Process Parameter Estimation." pp. 11431148.

[6] T. S. Perry, "Lotfi A. Zadeh [fuzzy logic inventor biography]," IEEE Spectrum, vol. 32, no. 6, pp. 32-35, 1995.

[7] D. Wilson, "Relay-based PID Tuning," Automation \& Control, pp. 10-12, 2005.

[8] L. A. Zadeh, "Fuzzy logic," Computer, vol. 21, no. 4, pp. 83-93, 1988.

[9] Zadeh, L., (1965) "Fuzzy logic", Fuzzy Sets and Systems, pp. 100-106. 